\title{
A New Treatment for the Reconstruction of the Medial Compartment of the Ankle: The Combined Use of Biological Materials
}

\author{
Marcello Lughi ${ }^{1}$ Alessandro Campagna ${ }^{1}$ Valeria Purpura ${ }^{2}$ Elena Bondioli ${ }^{2}$ \\ ${ }^{1}$ Orthopedics and Traumatology Operative Unit, Morgagni Hospital, \\ AUSL della Romagna, Forli, Italy \\ 2 Burn Center and Emilia Romagna Regional Skin Bank, Bufalini \\ Hospital, AUSL della Romagna, Cesena, Italy \\ Address for correspondence Valeria Purpura, PhD, Burn Center and \\ Emilia Romagna Regional Skin Bank, Bufalini Hospital, AUSL della \\ Romagna, Piazza della Liberazione 60, Cesena 47522, Italy \\ (e-mail: valeria.purpura@auslromagna.it).
}

Joints 2019;7:228-232.

\begin{abstract}
Fractures, especially if articular and periarticular, are frequently associated to functional and clinical disabling outcomes and chronic pain. In particular, the injuries with loss of bone, ligament, and/or tendon tissue in which the full recovery of the wound area is not obtained are the worst anatomical/pathological conditions to heal. In this study, three different biological materials were used as regenerative approaches to rebuild the medial malleolus fracture of the ankle in which loss of bone, ligament, and tendon tissue occur. In particular, the morselized human bone tissue was combined with the human dermis decellularized, both augmented with homologous platelet-rich

Keywords

- ankle

- reconstruction

- biomaterials

- fractures plasma. The magnetic resonance imaging study with contrast at the follow-up showed a signal compatible with vascularization of the tissue without sign of degeneration. Our new regenerative approach in which different biological materials were combined together showed a good choice of treatment for the reconstruction of not repairable outcome of a fracture.
\end{abstract}

\section{Introduction}

The treatment of fractures could be a challenge for surgeon especially when loss of bone, ligament, and/or tendon tissue occur. In the few past years the use of regenerative medicine approaches in these clinical conditions is increasing for their reconstructive/regenerative ability. To date, several biological materials are distributed by tissue banks for tissue reconstruction. In particular, the biological effectiveness of the skeletal muscle tissue and its derivatives depend on their ability to promote osteogenic, osteoinductive, and osteoconductive properties. ${ }^{1-4}$ Other biological materials frequently used alone as reconstructive/regenerative solution in different clinical conditions are the biological, cell-free matrices obtained

received

May 8, 2019

accepted after revision

April 18, 2021

published online

June 18, 2021 from decellularization of dermis in which the structural integrity remains unaltered. ${ }^{5}$ The platelet-rich plasma (PRP), in prevalence autologous, is also frequently used to enhance the regenerative action and the healing process on the lesion area.

The purpose of this study was the use for the first time of these three biological materials combined together as an innovative approach for the treatment of the same, not repairable lesion.

\section{Case Report}

A 38-year-old female patient showed at X-ray a pluriframmentary medial malleolus of the ankle and fractures to the

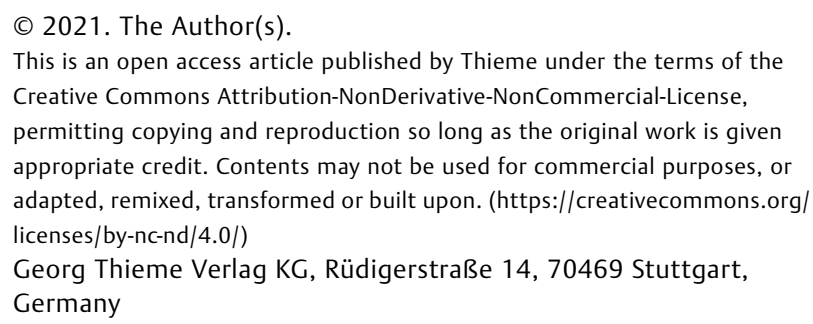




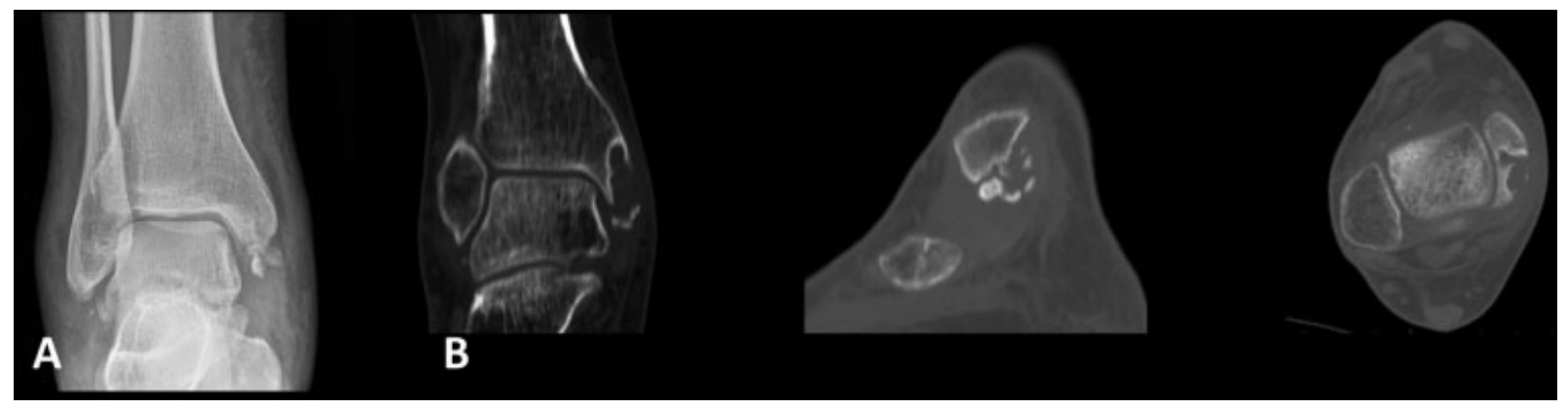

Fig. 1 (A) X-ray of the right ankle. Fracture of the medial malleolus. (B) Computerized tomography (CT) scans: "Cystic-like" lesion of the medial malleolus and bone fragments.

omolateral ileo and ischio pubic branches due to an incident that occurred 8 months before our observation (-Fig. 1A). The injury was conservatively treated with a nonweightbearing plaster boot for 30 days. After this period, the patient showed dry ankle, no pain, and no limitation in movement, and as a result functional rehabilitation was prescribed. During convalescence, the patient complained about constant pain, impossibility to weight-bearing, fixed foot in pronation, severe ankle limitation, and intense pain at the medial retromalleolar district palpation. The ankle appeared progressively more swollen especially on the medial segment and tests to assess the ankle and subtalar laxity were not performed due to intense pain. Computerized tomography (CT) scans of the ankle highlighted a "cystic-like" area with loss of bone tissue without an apparent containment wall at the level of the posterior part of the medial malleolus and the presence of numerous perimalleolar, probably mo-

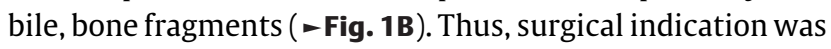
considered and reconstructive/regenerative procedures were chosen to treat bone loss, deltoid ligament injuries, and "pain" in the retromalleolar aspect of the ankle. To have a direct view of the medial compartment of the right ankle, the patient was placed in supine decubitus, with an ipsilateral hip extra rotated and with the knee flexed. Moreover, we applied a tourniquet at the root of the limb, while the contralateral limb was raised and sustained by a special support. Then, we performed a posterior medial incision of the skin, arched on the malleolus that was extended distally to the spring ligament complex and reached the bone plane, the ligament, and the posterior tibial tendon (-Fig. 2A). As a first step, we evaluated and treated the bone lesion: no solution of cortical was evident so that the cystic cavity, found on CT, was delimited by a continuous bone cortex but of reduced thickness and therefore easily penetrable by a series of needles that were used to "delimit" the perimeter. We then exposed the cystic cavity with an osteotome. Then, we filled the lesion area with the morselized bone allograft augmented with homologous PRP (-Fig. 2B). The portion of peripheral cortical was recomposed and stabilized with two titanium screws $1.7 \mathrm{~mm}$ in diameter $(\boldsymbol{-}$ Fig. $\mathbf{2 C}$ ). After that, we removed mobile and large perimalleolar bone fragments and, as a consequence, portions of ligament tissue. Then, the gap was quantified, without possibility of repair. We have therefore decided to rebuild the anteroinferior portion of the deltoid ligament using decellularized human dermal matrix (DHDM), $5 \times 5 \mathrm{~cm}$ in dimensions, that is produced by Emilia Romagna Regional Skin Bank through the application of a patented method of decellularization (PTC/ IB2008/002753). ${ }^{5}$ DHDM was sutured distally and marginally "side-to-side" to the ligamentous residue, while the remaining portion of it was sutured to the periost of the malleolus that was left intact proximally ( - Fig. $\mathbf{3 A - C}$ ). The DHDM under tension was then infiltrated with homologous PRP using an insulin needle. Then, we quantified the lesion of the posterior tibial tendon that showed a severe fibrillar structural alteration without loss of continuity. After the removal of the degenerated tendon tissue, we performed a tubulization wrapping the degenerated tendon with DHDM ( Fig. 4A, B), so that a "sealed" chamber around the tendon could be created and PRP was injected inside it ( $\mathbf{- F i g . ~ 4 C ) . ~}$ The ankle was immobilized with a 90-degree walker and weight-bearing was proscribed for approximately 35 days. After 15 days, a passive range of motion (ROM) was guaranteed by continuous passive motion of the ankle only in

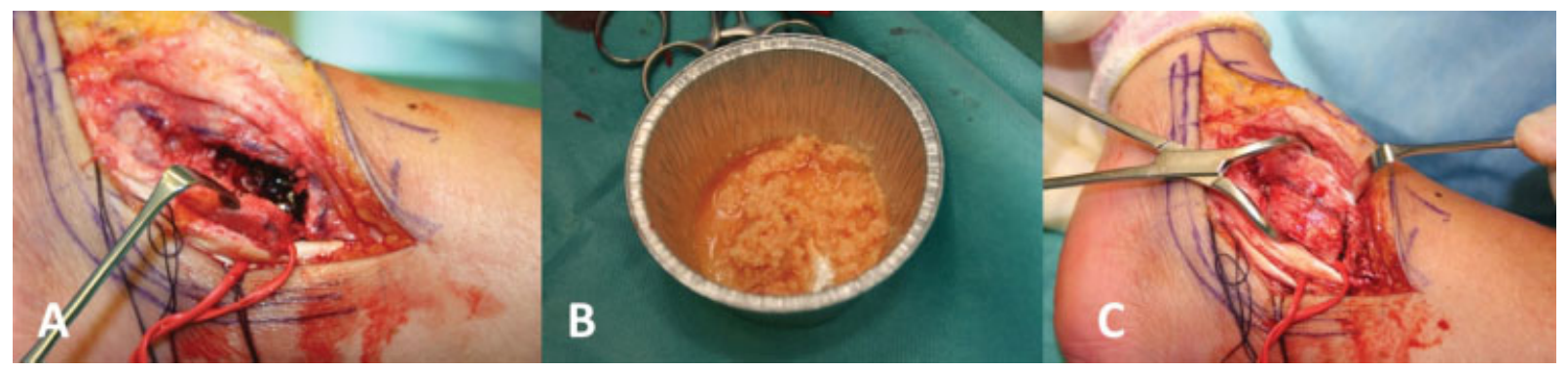

Fig. 2 (A) Open "cystic-like" medial malleolus. (B) Morselized bone allograft. (C) Closure of the cavity after boring. 


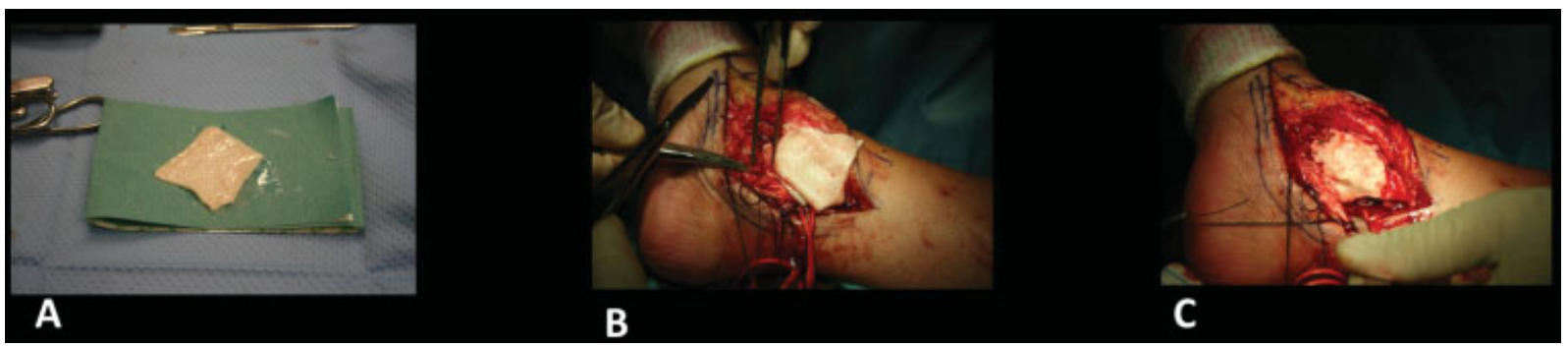

Fig. 3 (A) Decellularized human dermal matrix (DHDM). (B, C) DHDM sutured.

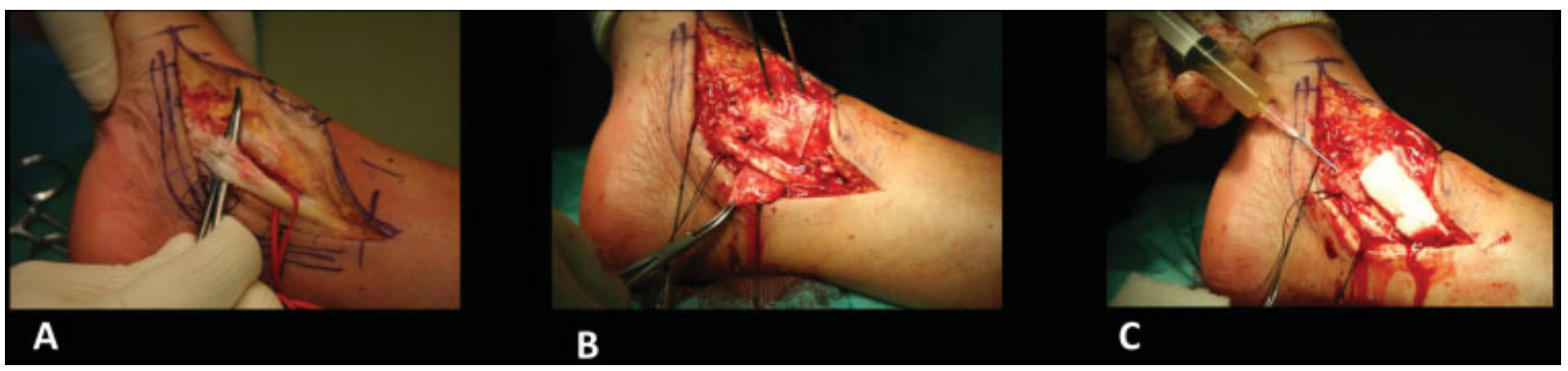

Fig. 4 (A) Posterior tibial tendon antomo-pathologic condition. (B) Decellularized human dermal matrix (DHDM) application around lesion. (C) Platelet-rich plasma (PRP) injection.

flexion-extension. After 35 days the patient started a program of articular and neuro- and myo-functional re-education. The clinical follow-up was then performed after 6 months. The magnetic resonance images (MRIs) showed a deltoid ligament and a posterior tibial tendon defined as normal (-Fig. 5B, D, yellow arrows). Moreover, the cystic bone bladder cavity was no longer evident. The patient recovered well, ankle was stable with normal function. To better identify the quality of the remodeled tissue, we performed an MRI with contrast after 9 months from intervention, since a biopsy sampling was not possible. The clinical results showed a signal compatible with vascularization (red arrow) of the tissue which is considered normal in the previous MRI without any sign of degeneration (yellow arrow) (-Fig. 5A, C, red arrows).

\section{Discussion}

In the last couple of years interest is increasing in the use of biological materials for the treatment of different clinical conditions, to take advantage of their regenerative potential. In particular, the use of bone tissue is widely useful in orthopaedics for the maintenance of its osteogenic, osteoinductive, and osteoconductive properties that makes it a suitable candidate to treat different orthopaedic conditions. ${ }^{1-4}$ Among the biological materials, the acellular dermal matrices are also particularly effective as biological cellfree scaffolds as they are currently widely used in different clinical fields for tissue reconstruction. ${ }^{6}$ For their effectiveness, biological materials are processed and distributed by tissue banks that are specific for different type of tissues. The autologous/homologous PRP as well as the mesenchymal cells derived from bone or adipose tissue are also frequently used in regenerative medicine. ${ }^{7}$ In particular, the high regenerative potential of homologous PRP distributed by transfusion service is suitable to enhance the healing process on the lesion area so that it is frequently used to induce regeneration. ${ }^{8,9}$ The clinical application of a single regenerative material as well as its use in combination with PRP was previously described in the literature. ${ }^{10-19}$ However, the level of evidence of published scientific works is not yet consistently high, so reported good clinical results are still of questionable statistical significance. In orthopaedics, the possible application of biological materials are numerous and topographically varied with the main purpose of solving problems of substance loss or tissue degeneration. Thus, an increase of scientific production about their use could be very helpful to improve treatments in this field.

With the aim to contribute with our experience to emphasize the effectiveness of biological materials in orthopaedics, here we reported results of a clinical case treated with three different biological materials in which loss of bone, ligament, and degenerative tendon tissue occur. To our knowledge, it is the first time that three different biological materials are combined together for the same patient appearing to be an innovative approach for the treatment of the same, not repairable lesion. In particular, we obtain morselized bone tissue by musculoskeletal tissue bank of Rizzoli Orthopedic Institute that has high expertise in the production of bone tissue for orthopaedic purposes. Moreover, we also used acellular dermal matrix developed and distributed by Emilia Romagna Regional Skin Bank of the AUSL Romagna. In particular, the skin tissue bank developed a patented decellularization method that is applied on human dermis to produce a dermal cell-free scaffold that previously showed excellent results in different clinical 


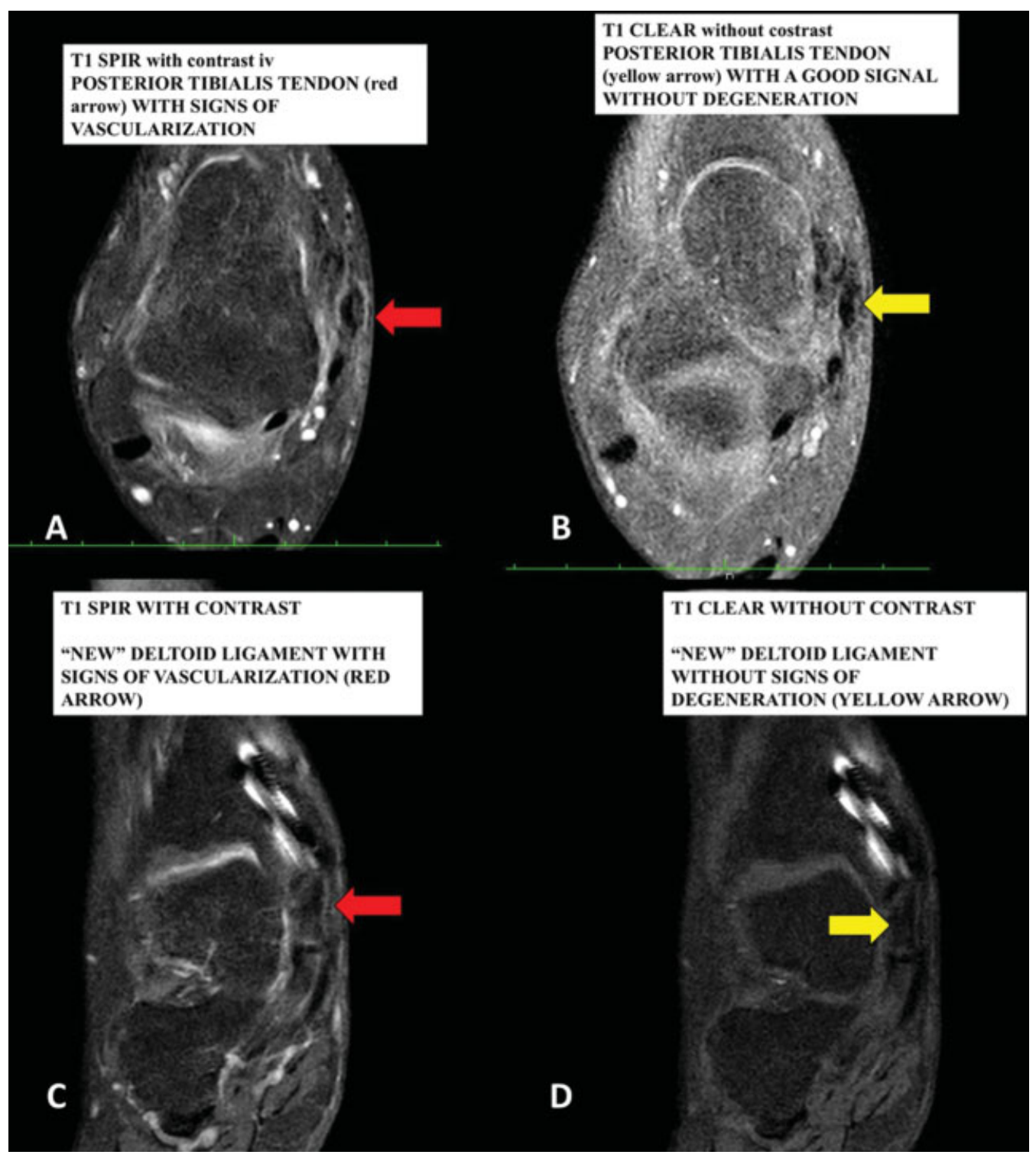

Fig. 5 Magnetic resonance imaging (MRI) with (A, C) or without (B, D) contrast intravenous at 6 and 9 months.

fields. ${ }^{20-24}$ In fact, the ability of DHDM to maintain a balance between structural integrity and the removal of cellular component makes it suitable for different type of tissue reconstruction. Thus, we decided to use the DHDM since it is able to mimic the structural and biological characteristics of tendons avoiding problems deriving from rejection. The PRP obtained by transfusion service of the AUSL Romagna was also used to give value to our treatment and to further promote the effectiveness of both biological materials and, in turn, the healing process. Although its use is still debated in literature, its effectiveness on bone tissue regeneration was previously described. ${ }^{13-17}$ Our clinical result was optimal considering that the patient has recovered the full ROM of the ankle joint, does not feel pain even after long periods of standing up for work, and does not report instability of the ankle and subtalar joints. Moreover, she can do any activity and reports improvements in her quality of life. From an instrumental point of view, the MRI with contrast medium has identified an optimal and "viable" regeneration of bone, tendon, and ligament structures. Thus, this case report show, even if not in a significant way, that the knowledge of the regenerative potential of different biological materials can determine the technical and surgical choices that are unique and also of high practical value. However, we have to consider that a potential drawback of the technique is the unpredictable evolution of the regeneration process. In fact, an evolution toward fibrosis instead of a finalistic anatomical recovery of the treated structures could have created the conditions for a rigid and nonfunctional ankle.

\section{Conclusion}

The combined regenerative approach using morselized bone tissue and DHDM, both infiltrated with homologous PRP in a "one-step" surgical procedure can be considered the first 
step of a new regenerative approach to the problems based on the use of regenerative medicine.

\section{Conflict of Interest}

None declared.

\section{Acknowledgments}

We thank Melandri D, MD, Director of Burn Center and Emilia Romagna Regional Skin Bank, AUSL della Romagna, Italy, for providing Decellularized Human Dermal Matrix. We thank Dallari D, MD, Director of Musculoskeletal Tissue Bank, Rizzoli Ortopedic Institute, AUSL of Bologna, Italy, for providing morselized bone tissue. We thank Agostini V, MD, Director of Immunohematology Service and Transfusion Medicine Operative Unit, AUSL della Romagna, Italy, for providing homologous PRP. We thank Bazzocchi O, MD, Radiologist of Morgagni Hospital, AUSL della Romagna, Italy, for providing MRI follow-up studies.

\section{References}

1 García-Gareta E, Coathup MJ, Blunn GW. Osteoinduction of bone grafting materials for bone repair and regeneration. Bone 2015; $81: 112-121$

2 Albrektsson T, Johansson C. Osteoinduction, osteoconduction and osseointegration. Eur Spine J 2001;10(Suppl 2):S96-S101

3 Fernandez de Grado G, Keller L, Idoux-Gillet Y, et al. Bone substitutes: a review of their characteristics, clinical use, and perspectives for large bone defects management. J Tissue Eng 2018;9:2041731418776819

4 Wang W, Yeung KWK. Bone grafts and biomaterials substitutes for bone defect repair: a review. Bioact Mater 2017;2(04):224-247

5 Bondioli E, Fini M, Veronesi F, et al. Development and evaluation of a decellularized membrane from human dermis. J Tissue Eng Regen Med 2014;8(04):325-336

6 Fosnot J, Kovach SJ III, Serletti JM. Acellular dermal matrix: general principles for the plastic surgeon. Aesthet Surg J 2011;31(7, Suppl):5S-12S

7 Han L, Fang WL, Jin B, Xu SC, Zheng X, Hu YG. Enhancement of tendon-bone healing after rotator cuff injuries using combined therapy with mesenchymal stem cells and platelet rich plasma. Eur Rev Med Pharmacol Sci 2019;23(20):9075-9084

8 Foster TE, Puskas BL, Mandelbaum BR, Gerhardt MB, Rodeo SA. Platelet-rich plasma: from basic science to clinical applications. Am J Sports Med 2009;37(11):2259-2272

9 Mariani E, Pulsatelli L. Platelet concentrates in musculoskeletal medicine. Int J Mol Sci 2020;21(04):E1328

10 Jones CP, Loveland J, Atkinson BL, Ryaby JT, Linovitz RJ, Nunley JA. Prospective, multicenter evaluation of allogenic bone matrix containing viable osteogenic cells in foot and/or ankle arthrodesis. Foot Ankle Int 2015;36(10):1129-1137

11 Hohn EA, Gillette BP, Burns JP. Outcomes of arthroscopic revision rotator cuff repair with acellular human dermal matrix allograft augmentation. J Shoulder Elbow Surg 2018;27(05):816-823

12 Acevedo DC, Shore B, Mirzayan R. Orthopedic applications of acellular human dermal allograft for shoulder and elbow surgery. Orthop Clin North Am 2015;46(03):377-388

13 Aspenberg P, Virchenko O. Platelet concentrate injection improves Achilles tendon repair in rats. Acta Orthop Scand 2004;75(01): 93-99

14 Smrke D, Gubina B, Domanoviç D, Rozman P. Allogeneic platelet gel with autologous cancellous bone graft for the treatment of a large bone defect. Eur Surg Res 2007;39(03):170-174

15 Bibbo C, Hatfield PS. Platelet-rich plasma concentrate to augment bone fusion. Foot Ankle Clin 2010;15(04):641-649

16 Wei LC, Lei GH, Sheng PY, et al. Efficacy of platelet-rich plasma combined with allograft bone in the management of displaced intra-articular calcaneal fractures: a prospective cohort study. J Orthop Res 2012;30(10):1570-1576

17 Bottegoni C, Dei Giudici L, Salvemini S, Chiurazzi E, Bencivenga R, Gigante A. Homologous platelet-rich plasma for the treatment of knee osteoarthritis in selected elderly patients: an open-label, uncontrolled, pilot study. Ther Adv Musculoskelet Dis 2016;8 (02):35-41

18 Jeong WH, Roh TS, Kim YS, et al. Acceleration of osteogenesis by platelet-rich plasma with acellular dermal matrix in a calvarial defect model. Childs Nerv Syst 2016;32(09):1653-1659

19 Çetiner D, Gökalp Kalabay P, Özdemir B, Çankaya ZT. Efficiency of platelet-rich plasma on acellular dermal matrix application with coronally advanced flap in the treatment of multiple adjacent gingival recessions: a randomized controlled clinical trial. J Dent Sci 2018;13(03):198-206

20 Melandri D, Marongiu F, Carboni A, et al. A new human-derived acellular dermal matrix for 1-stage coverage of exposed tendons in the foot. Int J Low Extrem Wounds 2020;19(01):78-85

21 Bondioli E, Purpura V, Orlandi C, et al. The use of an acellular matrix derived from human dermis for the treatment of fullthickness skin wounds. Cell Tissue Bank 2019;20(02): 183-192

22 Folli S, Curcio A, Melandri D, et al. A new human-derived acellular dermal matrix for breast reconstruction available for the European market: preliminary results. Aesthetic Plast Surg 2018;42(02): 434-441

23 Ghetti M, Bondioli E, Purpura V, Cenacchi G, Ruscelli P, Melandri D. Decellularized human dermal matrix produced by a skin bank: a new treatment for abdominal wall defects. Ann Ital Chir 2017; 5:443-448

24 Rotini R, Marinelli A, Guerra E, et al. Human dermal matrix scaffold augmentation for large and massive rotator cuff repairs: preliminary clinical and MRI results at 1-year follow-up. Musculoskelet Surg 2011;95(Suppl 1):S13-S23 